Neurosurg Focus 11 (6):Article 6, 2001 Click here to return to Table of Contents

\title{
Image-guided radiosurgery in the treatment of spinal metastases
}

\author{
Martin J. Murphy, Ph.D., Steven Chang, M.D., Iris Gibbs M.D., Quynh-Tu Le, M.D., \\ David Martin, M.D., AND Daniel Kim, M.D. \\ Departments of Neurosurgery and Radiation Oncology Stanford University School of Medicine, \\ Stanford, California
}

\begin{abstract}
Object. The authors describe a new method for treating metastatic spinal tumors in which noninvasive, image-guided, frameless stereotactic radiosurgery is performed. Stereotactic radiosurgery delivers a high dose of radiation in a single or limited number of fractions to a lesion while maintaining delivery of a low dose to adjacent normal structures.

Methods. Image-guided radiosurgery was developed by coupling an orthogonal pair of real-time x-ray cameras to a dynamically manipulated robot-mounted linear accelerator that guides the radiation beam to treatment sites associated with radiographic landmarks. This procedure can be conducted in an outpatient setting without the use of framebased skeletal fixation. The system relies on skeletal landmarks or implanted fiducial markers to locate treatment targets. Four patients with spinal metastases underwent radiosurgery with total prescription doses of 1000 to $1600 \mathrm{cGy}$ in one or two fractions. Alignment of the treatment dose with the target volume was accurate to within $1.5 \mathrm{~mm}$. During the course of each treatment fraction, patient movement was less than $0.5 \mathrm{~mm}$ on average. Dosimetry was highly conformal, with a demonstrated ability to deliver $1600 \mathrm{cGy}$ to the perimeter of an irregular target volume while keeping exposure to the cord itself below $800 \mathrm{cGy}$.

Conclusions. These experiences indicate that frameless radiosurgery is a viable therapeutic option for metastatic spine disease.
\end{abstract}

\section{KEY WORDS • frameless stereotaxis • radiosurgery • metastasis • spine}

Spinal tumors comprise $15 \%$ of all central nervous system neoplasms. The most common spinal lesions include metastatic disease, neurofibromas, schwannomas, meningiomas, lymphomas, myelomas, and astrocytomas, and arteriovenous malformations. ${ }^{1,24}$ Spinal metastases comprise the majority of tumors and affect more than 100,000 individuals yearly. ${ }^{3,4,20,21}$

The use of radiotherapy to treat metastatic cancer of the spine has been well established, ${ }^{10}$ but the spinal cord has a low threshold for radiation. Radiation-induced myelopathy can occur whenever the spinal cord is in the field of a radiation beam. Clinically, although the estimated risk of spinal cord injury is less than $0.2 \%$ for conventional doses of $45 \mathrm{~Gy}$ in 22 to 25 fractions, ${ }^{17}$ this sensitivity limits doses to levels that often fall short of optimum control. The spinal cord also appears to be more sensitive to larger doses per fraction. This is evidenced by an increase in the number of clinical reports in which myelopathy has been documented in cases receiving $40 \mathrm{~Gy}$ in four Gy fractions, ${ }^{17}$ compared with conventional doses. Radiotherapy can also cause spinal cord edema, which, although it

Abbreviations used in this paper: $\mathrm{CT}=$ computerized tomography; LINAC $=$ linear accelerator; $\mathrm{VB}=$ vertebral body. may resolve after completion of therapy, has an onset of symptoms that is insidious and often mimics epidural metastases. ${ }^{22}$

If the radiation dose can be confined more precisely to the treatment volume, then the likelihood of tumor control should increase at the same time that spinal cord injury is mitigated. Conventional external-beam radiotherapy, however, typically relies on manual positioning of the patient, which lacks the accuracy to allow delivery of large radiation doses to targets near radiosensitive structures. Furthermore, lesions along the spine can have especially complex topology as they infiltrate the bony structures, ${ }^{6}$ making conformal dose delivery difficult. Consequently, attempts to increase doses while protecting the spinal cord by using conformal radiotherapy have met with only limited success. ${ }^{7}$ This has led to a growing interest in applying stereotactic radiosurgery in the treatment of spinal lesions.

Lars Leksell developed the concept of stereotactic radiosurgery by combining stereotactic localization with multiple cross-fired beams from a collimated radiation source. ${ }^{12}$ This was intended to allow delivery of a very high dose of radiation to a treatment site while keeping the exposure of healthy tissue at a safe level. In conventional stereotactic radiosurgery a rigid frame is used to immobi- 
lize the lesion at a known location in space. The frame also acts as a fiducial reference system to provide accurate targeting and delivery of the radiation dose. Frame-based fixation has been shown to limit dose-alignment error to 1 to $2 \mathrm{~mm}$.

Surface doses of 12 to 20 Gy are typically used in the radiosurgical treatment of benign and malignant neoplasms. It has been observed that for fractions greater than 2 Gy, the LQ model for biological equivalence accounts for observations and that an $\alpha / \beta$ for the spinal cord is in the range of 2 to $2.5 \mathrm{~Gy} .{ }^{17}$ This provides a basis for estimating the extent to which the spine can tolerate large hypofractionated doses and, when combined with a measure of lesion proximity to the cord, sets a goal for dose alignment accuracy.

Because of the steep falloff in dose achieved with radiosurgery, and the anatomical relationship between many spinal vertebral lesions and the spinal cord, the spinal cord radiation dose can be quite low, provided it is accurately aligned. Hamilton, et al., ${ }^{8}$ for example, using a skeletal fixation method for spinal irradiation, determined that when administering an 8 to $15-$ Gy dose to a vertebral metastatic lesion, the median spinal cord radiation exposure was approximately $8 \%$ of the prescribed dose. Although this may be achievable in cases of extramedullary lesions, which account for most primary tumors of the spine, intramedullary lesions present a greater challenge. In this case, fractionation of the dose is advisable to ensure that exposure of the cord remains within a safe range. Therefore, whereas spinal radiosurgery offers potential therapeutic advantages, it must be delivered with high accuracy and conformity while simultaneously allowing for fractionation.

Spinal lesions have a fixed relationship to the spine, which has led to the development of a radiosurgical frame that attaches to the VBs. ${ }^{6,9}$ This system, however, is invasive and requires either local or, more commonly, general anesthesia. Additionally, because reattachment of such a frame is problematic, it would need to be left in place to fractionate the dose. An alternative system for use in spinal radiosurgery is an external stereotactic frame that encloses the body from head to the middle thigh area and stabilizes the patient by using a foam pad within the frame. ${ }^{11}$ Because this system does not provide rigid fixation, however, it allows for some residual patient movement.

Until recently, radiosurgery has been performed using a spherical configuration of fixed radiation sources or a gantry-mounted LINAC, each producing cylindrical pencil beams. Both radiosurgical options produce spherical dose distributions with isocenters fixed in relation to the radiation source. More complex dose distributions are approximated by overlapping multiple spheres, which requires shifting the patient with respect to the beam isocenter and results in dose inhomogeneities within the treatment volume. Radiation treatment of structures near the spinal cord would benefit from a technique that can conform to the tumor shape while maintaining dose homogeneity and sparing the spinal cord.

Image-guided frameless stereotactic radiosurgery was developed as a noninvasive means to align and conform treatment doses to target volumes precisely. It has three fundamental differences from conventional frame-based radiosurgery. First, it references the position of the treatment site to internal radiographically documented features, such as skeletal anatomy or implanted fiducials, rather than a frame. Second, it uses real-time radiographics to establish the position of the lesion during treatment and then brings the radiation beam into alignment with the observed position of the treatment site. Changes in patient position during treatment are accommodated by adaptive beam pointing rather than being accepted as a setup error or controlled through rigid immobilization. This ability to compensate for patient-related movement allows the patient to be positioned in the treatment room without having to reproduce precisely the position in the treatment planning study. Third, it aims each beam independently, without a fixed isocenter, which enables formation of irregular dose shapes with good homogeneity.

Frameless image-guided radiosurgery ${ }^{2}$ using the CyberKnife has recently been introduced for treatment of the spine. ${ }^{14,15}$ In this procedure, the position of the treatment site is referenced to radiographically determined features such as the VBs or implanted fiducials. The CyberKnife consists of a 6-MV LINAC mounted on a robotic arm that is coupled through a control loop to an x-ray imaging system. During treatment the imaging system acquires radiographs of the targeting landmarks. The landmarks are registered to the treatment-planning CT study to determine the lesion's position. The observed position is transmitted to the robot, which then aligns the radiation beam with the treatment site. The entire targeting process-image acquisition, processing, registration, and beam realignment - is completely automatic and fast enough to be performed repeatedly during treatment. Changes in patient position are compensated by adaptive beam pointing rather than controlled through immobilization or being accepted as setup errors. This procedure facilitates fractionation and enables high precision in dose placement, assuming that the tumor maintains a fixed relationship with the radiographic targeting features and that the patient does not move appreciably during treatment.

At Stanford University the CyberKnife has been used to treat 25 cases of fractionated spine radiosurgery in sites ranging from $\mathrm{C}-1$ to $\mathrm{S}-1$. In four of these cases the patients received treatment for metastatic thoracic and lumbar tumors. All procedures were performed on an outpatient basis. Targeting of the cervical spine was based on vertebral landmarks. The thoracic and lumbar sites, which are more difficult to image and register, were marked by fiducials implanted in nearby VBs. These experiences have shown that dose alignment can meet the radiosurgical standard of 1- to 2-mm precision, patient mobility is minimal, and dose planning meets the accepted standards for isocentric radiosurgery. Because the use of frameless spinal radiosurgery has been demonstrated to be feasible, we introduce this new therapeutic option for metastatic spinal disease.

\section{CLINICAL MATERIAL AND METHODS Patient Population}

Four patients with metastases to the spine were treated using CyberKnife stereotactic radiosurgery between 1999 
and 2000. The mean patient age was 64.8 years (range 54-73 years). There were three women and one man. In each patient the primary disease was different: esophageal cancer, melanoma, renal cell cancer, and breast cancer. Two patients harbored thoracic and two lumbar lesions. In the two cases in which the thoracic spine was affected, both patients harbored two lesions. The thoracic lesions were treated in two fractions; the lumbar lesions were treated in a single fraction.

\section{Treatment Planning}

The treatment dose for the six lesions varied between 1040 and $1600 \mathrm{cGy}$ (mean $14.1 \mathrm{cGy}$ ), which was prescribed to the 70 to $80 \%$ isodose contour at the edge of target volume. The maximum intratumoral dose ranged from 1500 to $2000 \mathrm{cGy}$ (mean $1725 \mathrm{cGy}$ ). A limit of 800 cGy was set as the maximum spinal cord dose. ${ }^{8}$ The dose to the tumor margin was based on volume, location, and history of fractionated radiotherapy. Secondary collimators ranged in diameter from 15 to $35 \mathrm{~mm}$. The tumor volumes ranged from 3.8 to $47.9 \mathrm{~cm}^{3}$. These cases are summarized in Table 1.

The CyberKnife treatment-planning system is a Food and Drug Administration-approved program that coordinates the radiation treatment plan with the mechanical delivery of radiation. Treatment planning proceeds in two steps. First, the process chooses a group of beam nodes from approximately 150 possible positions. The beam nodes consist of static positions for the LINAC arranged around an anulus of radius $80 \mathrm{~cm}$ and centered at the treatment volume. Guided by a prescribed dose distribution and avoidance constraints for critical structures, the system adjusts beam directions and dose intensities (beam weights) for each node until optimum conformity is reached with the prescribed distribution. The beam directions are not confined to a common isocenter. ${ }^{18}$ In this fashion, the CyberKnife not only can emulate a gantry LINAC and spherical gamma knife but can also produce doses with ellipsoidal, toroidal, and other complex shapes. Careful planning can produce beam distributions that wrap around the spine, ensuring nominal exposure to the cord.

\section{Image Guidance}

At Stanford the CyberKnife has been specially adapted to perform a variety of extracranial procedures. It uses flat-panel amorphous silicon x-ray cameras (dpiX LLC, Palo Alto, CA $)^{25}$ for patient positioning and tracking. These devices have $19.2 \times 24-\mathrm{cm}$ active areas and a pixel

\section{TABLE 1}

Summary of treatment data in four patients undergoing image-guided radiosurgery

\begin{tabular}{cllll}
\hline \hline $\begin{array}{c}\text { Case } \\
\text { No. }\end{array}$ & $\begin{array}{l}\text { Primary } \\
\text { Disease }\end{array}$ & $\begin{array}{c}\text { Site of } \\
\text { Metastases }\end{array}$ & $\begin{array}{c}\text { Volume } \\
\left(\mathrm{cm}^{3}\right)\end{array}$ & Dose (cGy) \\
\hline 1 & breast & L-4 & 47.9 & $1600 @ 80 \%$ \\
2 & esophageal & L-1 & 3.8 & $1500 @ 75 \%$ \\
$3 *$ & melanoma & T-1/T-5 & 0.88 & $1040 / 1700 @ 80 \%$ \\
$4 *$ & renal cell & T-4/T-6 & 6.9 & $1200 / 1200 @ 80 \%$ \\
\hline
\end{tabular}

* Each patient harbored two lesions. pitch of $0.125 \mathrm{~mm}$. They acquire flat images that avoid the distortions inherent in lensed or x-ray image-intensifier cameras. They have sufficient sensitivity to detect radiographic features in the neck, thorax, and abdomen. The imaging system has been designed to provide variable fields of view and magnification to accommodate both large and small targeting landmarks. The Stanford CyberKnife system also uses an advanced image registration process that recognizes a variety of radiographic landmarks and measures all six degrees of freedom in the position and orientation of patient anatomy. ${ }^{13}$ These modifications allow the system to target lesions along the spine and in abdominal soft-tissue sites. ${ }^{5,14}$

The central function of the image guidance process is to measure changes in the position of the target site during treatment, compared with its position in the planning study. Once these changes have been determined, the originally planned beam directions can be realigned to the new position of the target. The position measurement is made first by acquiring two radiographs of the targeting landmarks, using the real-time x-ray cameras in the treatment room. A sequence of digitally reconstructed radiographs is then calculated in real time from the planning study, imitating the viewing position of the camera system. The anatomical position demonstrated in the CT study is changed iteratively until the digitally reconstructed radiographs match the acquired radiographs. The perturbation in position that produces matching digitally reconstructed radiographs and live images corresponds to the change in beam directions needed to direct the beam at the observed target position. This process accommodates both the intrinsic variability in patient setup position at the beginning of treatment and any subsequent changes caused by patient movement during treatment.

\section{Treatment Delivery}

A contrast-enhanced CT scan of the region of interest was acquired in each patient for treatment planning and also for point of reference in the image-guidance process. In some patients, magnetic resonance imaging was also performed and the images then fused with the CT scans to create a composite imaging study.

Thoracic and lumbar vertebrae, which are difficult to image with the present CyberKnife system, were marked with small implanted fiducials to provide enhanced imaging landmarks. After induction of conscious sedation, four $2 \times 6-\mathrm{mm}$ surgical stainless-steel self-retaining tacks were placed through skin incisions under fluoroscopy in a noncoplanar pattern on the lamina or facet joint around the lesion of interest. No procedure-related complications were demonstrated, and all patients were discharged home on the same day. ${ }^{15}$

All patients were treated in the supine position while resting in a comfortable alpha cradle to minimize body movement. For each fraction the dose was divided among 100 or more beam directions (that is, nodes). In the treatment of these six spinal tumors, the beam directions were arranged in an annulus with the axis along the spine. During treatment the robotic arm moved the LINAC sequentially through the prescribed beam positions. At each position at which the LINAC stopped, the imaging system had the opportunity to check the target position and send cor- 
rective pointing directions to the robot, and the radiation beam was then turned on for the prescribed duration. The LINAC was moved to the next beam position and the process was repeated. The position of the treatment site was checked at approximately 1-minute intervals throughout each treatment fraction. This enabled adaptation to the small and infrequent position shifts of the patient, which were documented in a record of the treatment site positions observed by the imaging system.

\section{RESULTS}

\section{Treatment Planning and Dosimetry}

Figure 1 shows three orthogonal views of the treatment plan for the patient in Case 1 who harbored a breast cancer metastatic tumor at L-4. The green curve marks a dose of $1600 \mathrm{cGy}$ at the $80 \%$ isodose line. This isodose line encloses $94 \%$ of the target volume. The magenta curve indicates a dose of $1000 \mathrm{cGy}$ at the $50 \%$ isodose line. The spinal cord was avoided, which kept the dose to the cord below 900 cGy throughout its volume. This plan has been evaluated quantitatively to judge its conformity with accepted radiosurgical standards.

Dosimetric standards for radiosurgery have evolved from the capabilities of the gamma knife. To inaugurate a multiinstitutional protocol to evaluate the effectiveness of radiosurgery, Shaw, et al., ${ }^{19}$ proposed a set of dosimetry indices to characterize radiosurgical treatment plans. They suggested numerical ranges for these indices that would characterize individual plans as 1) being fully within the standards associated with radiosurgery or 2) deviating in a minor or a major way from those standards. The three dosimetry indices presented by Shaw, et al., are based on the prescription and actual target isodose curves, where the actual target isodose is defined as the curve that mini- mally encloses the entire target volume. The three indices originally defined by Shaw, et al., are as follows.

Coverage Index. The coverage index is the target isodose, as a fraction of the prescription isodose. If the isodose line corresponding to $90 \%$ of the prescribed dose completely encloses the target, the plan is considered to follow protocol. If less than $90 \%$ but more than $80 \%$ of the prescribed dose completely encloses the target, the plan is considered a minor deviation. In all other variations, it is a major deviation.

Homogeneity Index. This is the ratio of the maximum dose to the prescription isodose. If it is less than 2 , the plan is per protocol; between 2 and 2.5, a minor deviation; and greater than 2.5, a major deviation.

Conformity Index. This is the ratio of the prescription isodose volume to the target volume (calculated from the integral dose volume histograms for the target volume and the normal tissue volume). If the ratio is between 1 and 2, the plan is per protocol between 2 and 2.5, a minor deviation; and greater than 2.5, major deviation.

Defined in relation to the aforementioned parameters, however, the indices are not internally self-consistent or fully descriptive of the quality of the plan. Homogeneity and conformity are better described using the actual target isodose rather than the prescription isodose, because for complex configurations of tumors and critical structures the prescription isodose cannot always be achieved (as is recognized by the coverage index). Furthermore, the original conformity criteria proposed by Shaw, et al., ${ }^{19}$ conflict with the coverage index. The coverage index allows for the prescription isodose surface to be partly or wholly within the tumor volume, such that the tumor volume is completely enclosed instead by, for example, $95 \%$ of the prescription isodose, and still be according to protocol. This situation could result in a conformity index less than

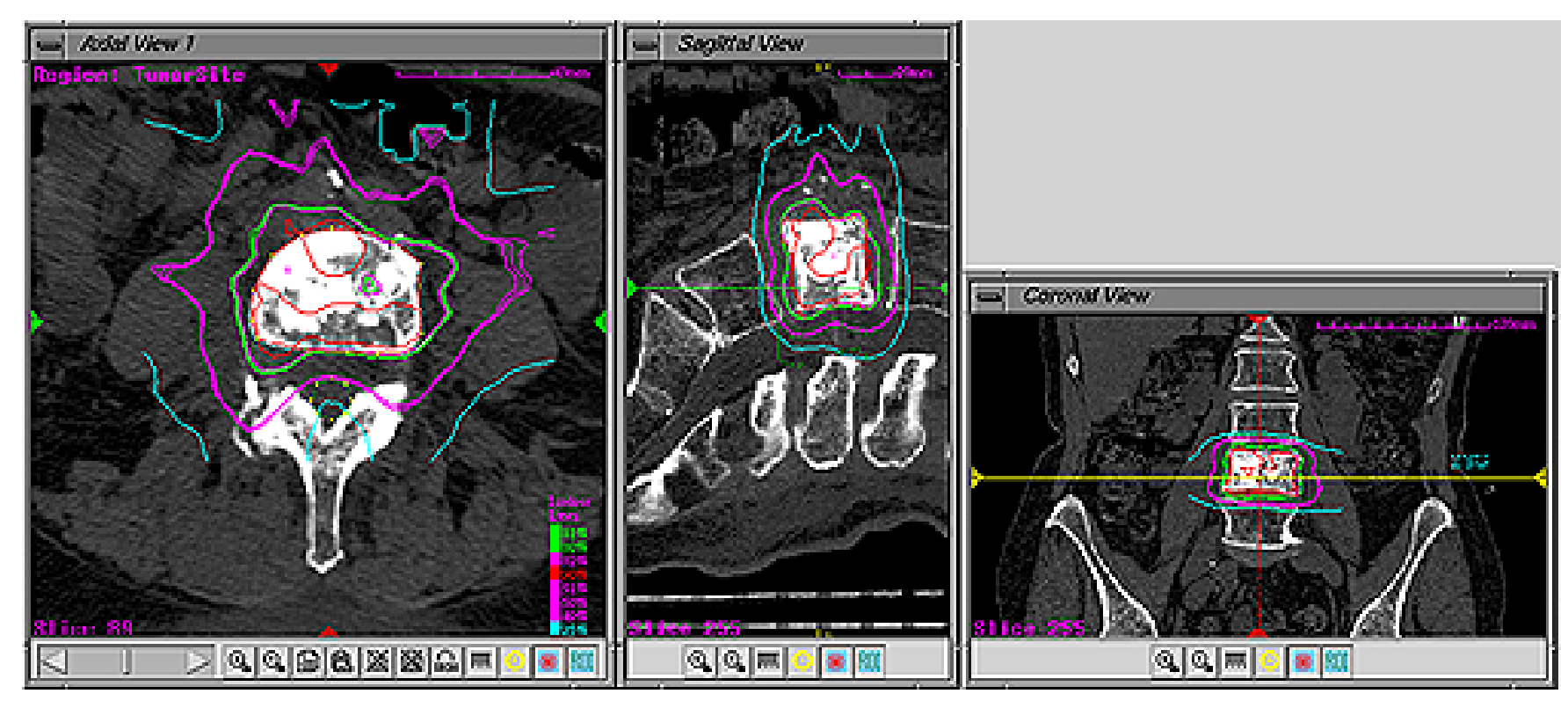

Fig. 1. Case 1. Axial (left), sagittal (center), and coronal (right) views of the planned dose distribution for an L-4 metastatic lesion. The green curve indicates the prescribed $80 \%$ isodose line, corresponding to $1600 \mathrm{cGy}$, and the magenta curve indicates the $50 \%$ isodose line, corresponding to $1000 \mathrm{cGy}$. 
1, however, which would classify it as a minor deviation. Furthermore, for irregular tumor shapes, the conformity index could be 1.0 for plans with poor conformality and poor coverage. Therefore, we compute the homogeneity index and the conformity index by using the delivered target isodose rather than the prescription isodose. This is in fact a more conservative assessment, because the coverage index is always expected to be less than or equal to 1 , so that using the target isodose rather than the prescription isodose actually increases the value of the homogeneity and conformity indices.

For the plan illustrated in Fig. 1, the coverage index is 0.95 , the homogeneity index is 1.32 , and the conformity index is 1.47 , making the plan fully compliant with the radiosurgery protocol. In nearly all of the spinal radiosurgical treatments undertaken to date, the treatment plans fully met all the criteria for the radiosurgery protocol. ${ }^{15}$

\section{Accuracy of Dose Delivery}

The accuracy of CyberKnife dose placement depends on the resolution of the treatment-planning CT study, the real-time registration between $\mathrm{CT}$ scans and treatment radiographs, and the pointing accuracy of the robot. (In frame-based LINAC radiosurgery the corresponding sources of error are treatment-planning resolution, frame flexure and distortion, and gantry rotation error.) In spinal radiosurgery, a CT slice of $1.25 \mathrm{~mm}$ was typically used, which introduced an uncertainty of $0.7 \mathrm{~mm}$ in the inferior/superior coordinate of the treatment volume. The pointing accuracy of the robot was approximately $0.7 \mathrm{~mm}$. Image registration based on fiducial landmarks was accurate to approximately $0.2 \mathrm{~mm}$, whereas error in vertebral landmark registration was approximately $0.5 \mathrm{~mm}$. These various components of system accuracy, and the combined overall accuracy, have been measured using imaging and dosimetry phantoms. Altogether, the root-meansquare targeting error was approximately $1.5 \mathrm{~mm}$.

Use of the alpha cradle minimized patient movement. Furthermore, by undertaking treatment with the patient supine, respiratory motion was minimal. Any residual movements were detected by the imaging system and corrected within 1 minute or less. Figure 2 illustrates the record of position measurements in one patient. The amount of residual movement can be characterized by the change in target position from one imaging measurement to the next. These changes were measured along each patient coordinate axis for all the treatments and collected into the histogram shown in Fig. 3. The mean fluctuation in patient position during successive position measurements was $0.3 \mathrm{~mm}$, and three fourths of the fluctuations were less than $0.5 \mathrm{~mm}$.

\section{DISCUSSION}

To apply radiosurgery to the spine successfully, three conditions must be met: 1) the radiation delivery system must be able to produce a highly conformal dose distribution that spares the spinal cord; 2) it must be able to target the dose distribution to the intended site with millimeterlevel precision; and 3) it must be capable of fractionated treatment delivery. Frameless image-guided radiosurgery has been developed to meet these requirements.

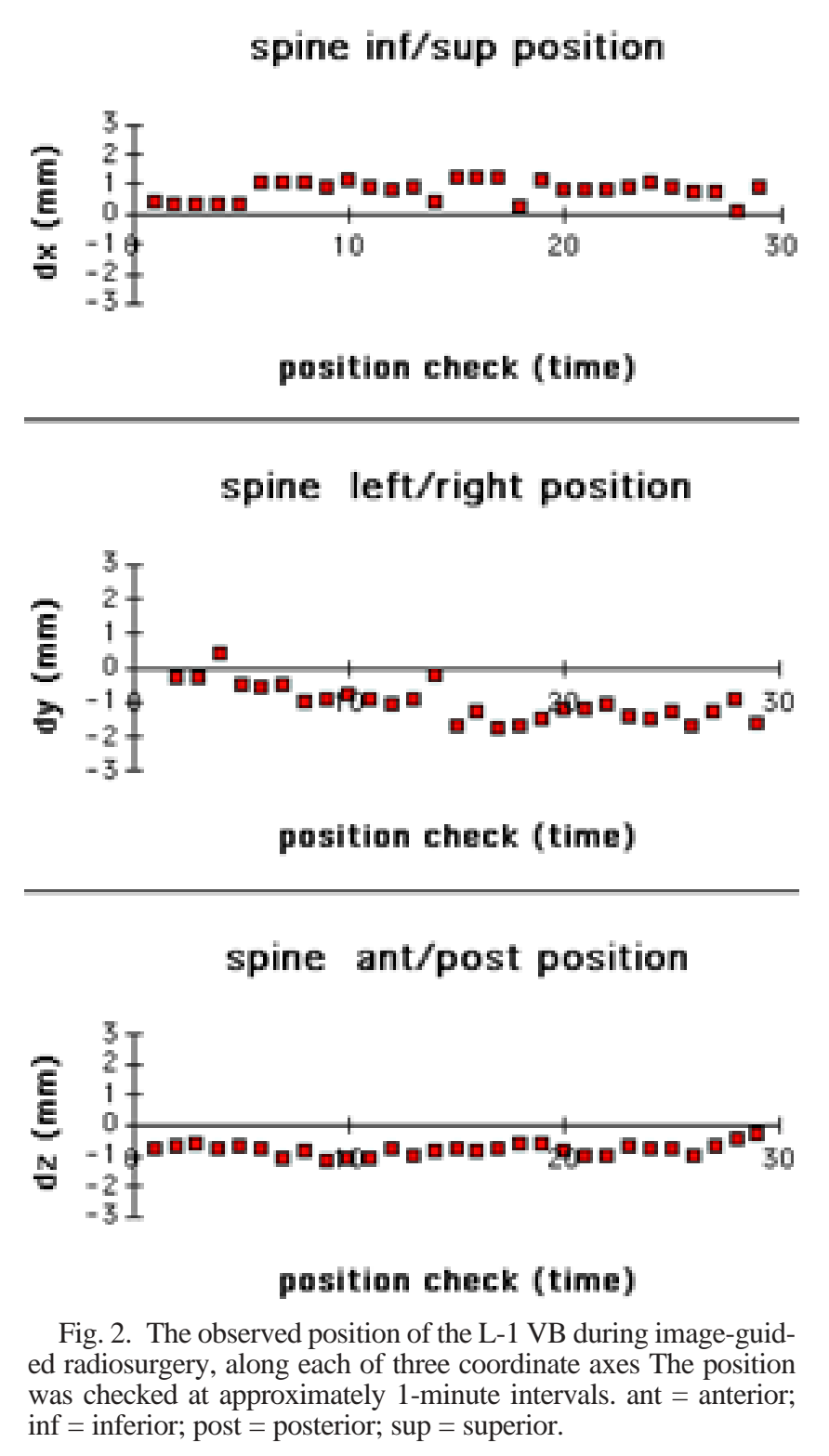

When guided by fiducials, dose placement error using the CyberKnife is on the order of $1.5 \mathrm{~mm}$, which includes all the uncertainties associated with defining the target volume coordinates on the $\mathrm{CT}$ study, positioning the patient by radiographic imaging, and directing the treatment beams robotically. This overall precision is in line with the generally accepted standard for radiosurgery. ${ }^{16}$

During treatment with the CyberKnife, the patient is neither immobilized nor continuously tracked in real time to maintain alignment of the radiation beam. If the patient makes frequent and significant movements (for example, repeatedly shifting $1 \mathrm{~mm}$ or more during the interval between image acquisitions of the target position), the accuracy of dose placement will be compromised. It was therefore important to observe the range and frequency of movement. By periodically rather than continuously reimaging the patient's position, one obtains a random sample of variations in the patient position; this sample is rep- 


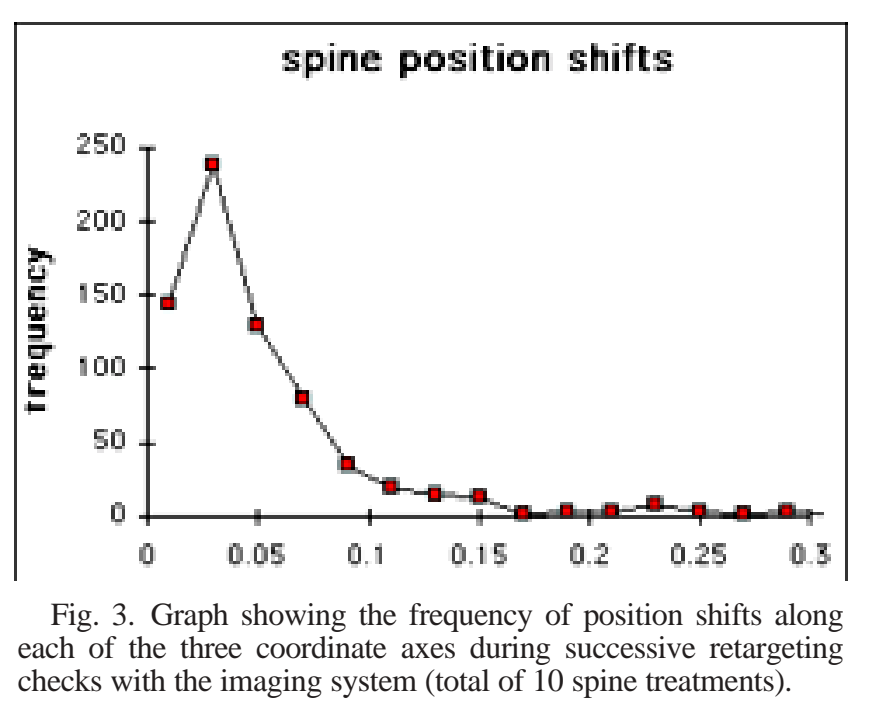

resentative of the patient's movement during treatment. In our experience, patients treated while supine in an alpha cradle remained essentially motionless (Fig. 3) Typically, only one or two shifts of more than $1 \mathrm{~mm}$ were observed during each 30-minute treatment fraction. Because the shifts occurred at random times while the beam was on, and going undetected until the next updated image is acquired, the result, on average, was that half the dose delivered between targeting corrections is misaligned. If 50 targeting corrections were made during a fraction, then approximately 1 to $2 \%$ of the dose was mistargeted by 1 $\mathrm{mm}$ or more. This was judged acceptable and formed a basis for setting the frequency of reimaging the patient's position during each fraction.

The use of artificial fiducials to enhance the visibility of vertebral landmarks provides a well-tolerated solution to targeting lesions in the thoracic and lumber spine, although it makes the procedure invasive. Ideally, frameless stereotaxy would rely solely on internal landmarks; however, although bone landmarks in the spine are suitable for image registration, they are not readily discerned in the thorax and abdomen when using the present CyberKnife imaging system. Until the imaging senstivity is improved, spine fiducials will continue to be required to supplement the guidance process.

The CyberKnife has nearly complete freedom in directing pencil beams of radiation at the lesion without the constraint of an isocenter. It has been suggested that this dose-delivery method could be the optimum strategy for obtaining highly conformal dose distributions. ${ }^{23}$ To exploit this strategy fully, the CyberKnife uses an inverse treatment-planning process. The lesion to be treated is outlined, and the desired dose is specified. Dose limits are specified to the surrounding sensitive structures such as the spinal cord. The treatment-planning station then automatically generates an optimized treatment plan of specific beam orientations and dose intensities. The resulting plan can deliver a more homogenous dose to the lesion than would be possible when using an isocenter-constrained system, and it is able to restrict high-dose radiation for radiosensitive structures such as the spinal cord. When using this kind of inverse process, however, it is important to define all of the dose regions that are significant; in the absence of an explicit constraint, the planning process will be free to direct beams through any part of the anatomy.

The images in Fig. 1 illustrate the ability to achieve good conformality while avoiding critical structures. The perimeter of the target volume is less than $1 \mathrm{~cm}$ from the spinal cord and follows the outline of the entire VB. The radiation dose to the target margin is $1600 \mathrm{cGy}$ while the maximum dose to the proximal edge of the cord is below 900 cGy.

The radiation doses that were used to treat our patients met the Radiation Therapy Oncology Group standards ${ }^{19}$ for radiosurgery, despite the irregular shape of the lesions and their extreme proximity to a major critical structure (the spinal cord) in all the cases. The homogeneity index in particular was always well within the protocol standard, which demonstrated that nonisocentric beam pointing can produce better homogeneity than a single-isocenter technique. This benefit arises mainly because the system can overlap beams that are significantly smaller in diameter than the cross section of the lesion, which reduces the effect of penumbra and the nonflat profile of small-diameter cylindrical beams.

During the evaluation of the spinal plans against the Radiation Therapy Oncology Group protocol standards, ${ }^{19}$ it became clear that the three original indices were tailored to the spherical isocentric dosimetry of conventional frame-based radiosurgery and did not do an effective job of characterizing highly conformal plans for complex configurations of lesions and critical structures. The original conformity index, in particular, was completely inadequate for any plan involving, for example, a highly elongated tumor, because it only required a match of isodose volumes while ignoring the orientation of the dose distribution with respect to the lesion. Additionally, the homogeneity criteria were too relaxed given the capabilities of contemporary radiation beam delivery systems. This has demonstrated the need for a new and more sophisticated way to evaluate spine radiosurgery plans.

\section{CONCLUSIONS}

Frameless robotic radiosurgery provides a new way to deliver accurate high-dose radiotherapy to lesions that are not amenable to conventional radiosurgery. This technology has been expanded to treat extracranial spinal cord lesions. Whether targeted on skeletal landmarks or percutaneously implanted fiducial markers, image-guided radiosurgery can achieve spatial accuracy comparable with frame-based cranial treatments without the inconvenience or limitations associated with frames. Its safety and efficacy appear to be similar to that of conventional radiosurgery while providing the flexibility of a less constrained treatment delivery system.

\section{References}

1. Adams JH, Graham DI, Doyle D: Brain Biopsy: The Smear Technique for Neurosurgical Biopsies. London: Chapman and Hall, 1981

2. Adler JR Jr, Murphy MJ, Chang SD, et al: Image-guided robotic radiosurgery. Neurosurgery 44:1299-1307, 1999 
3. Black P: Spinal metastasis: current status and recommended guidelines for management. Neurosurgery 5:726-746, 1979

4. Byrne TN, Waxman SG: Spinal Cord Compression: Diagnosis and Management. Philadelphia: Davis, 1990, p 147

5. Chang,SD, Adler JR Jr, Murphy MJ: Stereotactic radiosurgery of spinal lesions, in Maciunas RJ (ed): Advanced Techniques in Central Nervous System Metastases. Park Ridge, IL: American Association of Neurological Surgeons, 1998, pp 269-276

6. Hamilton AJ: Stereotactic radiosurgery of spinal metastases, in Maciunas RJ (ed): Advanced Techniques in Central Nervous System Metastases. Park Ridge, IL: American Association of Neurological Surgeons, 1998, pp 255-268

7. Hamilton AJ, Lulu B, Stea B, et al: The use of gold foil wrapping for radiation protection of the spinal cord for recurrent tumor therapy. Int J Radiat Oncol Biol Phys 32:507-511, 1995

8. Hamilton AJ, Lulu BA, Fosmire H, et al: LINAC-based spinal stereotactic radiosurgery. Stereotact Funct Neurosurg 66: $1-9,1996$.

9. Hamilton AJ, Lulu BA, Fosmire H, et al: Preliminary clinical experience with linear accelerator-based spinal stereotactic radiosurgery. Neurosurgery 36:311-319, 1995

10. Kopelson G, Linggood RM, Kleinman GM, et al: Management of intramedullary spinal cord tumors. Radiology 135:473-479, 1980

11. Lax I, Blomgren H, Naslund I, et al: Stereotactic radiotherapy of malignancies in the abdomen. Methodological aspects. Acta Oncol 33:677-683, 1994

12. Leksell L: The stereotactic method for radiosurgery of the brain. Acta Chir Scand 102:316-319, 1951

13. Murphy MJ: An automatic six-degree-of-freedom image registration algorithm for image-guided frameless stereotaxic radiosurgery. Med Phys 24:857-866, 1997

14. Murphy MJ, Adler JR Jr, Bodduluri M, et al: Image-guided radiosurgery for the spine and pancreas. Comput Aided Surg 5: 278-288, 2000

15. Ryu SI, Chang SD, Kim DH, et al: Image-guided hypo-fractionated stereotactic radiosurgery to spinal lesions. Neurosurgery 29:838-846, 2001

16. Schell MC, Bova FJ, Larson DA, et al: Stereotactic Radiosur- gery: Report of Task Group 42 Radiation Therapy Committee. College Park, MD: American Institute of Physics, 1995, pp 6-8

17. Schultheiss TE, Kun LE, Ang KK, et al: Radiation response of the central nervous system. Int J Radiat Oncol Biol Phys 31: 1093-1112, 1995

18. Schweikard A, Adler JR, Latombe JC: Motion planning in stereotaxic radiosurgery. IEEE Trans Rob Autom 9:764-774, 1993

19. Shaw E, Kline R, Gillin M, et al: Radiation Therapy Oncology Group: radiosurgery quality assurance guidelines. Int J Radiat Oncol Biol Phys 27:1231-1239, 1993

20. Smaltino F, Bernini FP, Santoro S: Computerized tomography in the diagnosis of intramedullary metastases. Acta Neurochir 52:299-303, 1980

21. Sundareson N, Krol G, DiGiacinto GV, et al: Metastatic tumors of the spine, in Sundareson N, Schmidek HH, Schiller AL, et al (eds): Tumors of the Spine: Diagnosis and Clinical Management. Philadelphia: WB Saunders, 1990, pp 279-304

22. van der Kogel AJ: Radiation injury in the central nervous system, in Alexander E III, Loeffler JS, Lunsford D (eds): Stereotactic Radiosurgery. New York: McGraw-Hill, 1993, pp 43-64

23. Webb S: Conformal intensity-modulated radiotherapy (IMRT) delivered by robotic linac--testing IMRT to the limit? Phys Med Biol 44:1639-1654, 1999

24. Weinstein JN, McLain RF: Tumors of the spine, in Rothman RH, Simeone FA (eds): The Spine. Philadelphia: WB Saunders, 1992, Vol 1, pp 1279-1318

25. Weisfield RL, Hartney MA, Schneider R, et al: High performance amorphous silicon image sensor for X-ray diagnostic medical imaging applications. SPIE 3659:307-317, 1999

Manuscript received October 22, 2001.

Accepted in final form November 14, 2001.

Address reprint requests to: Martin J. Murphy, Ph.D., Department of Radiation Oncology A-044, Stanford University School of Medicine, 300 Pasteur Drive, Stanford, California 94305. email: martin@ reyes.stanford.edu. 